\title{
M-ary Aggregate Spread Pulse Modulation in LPWANs for loT applications
}

This paper was downloaded from TechRxiv (https://www.techrxiv.org).

LICENSE

CC BY-NC-SA 4.0

SUBMISSION DATE / POSTED DATE

31-08-2021 / 05-09-2021

CITATION

Nikitin, Alexei; Davidchack, Ruslan (2021): M-ary Aggregate Spread Pulse Modulation in LPWANs for loT applications. TechRxiv. Preprint. https://doi.org/10.36227/techrxiv.16550088.v1

DOI

10.36227/techrxiv.16550088.v1 


\section{M-ary Aggregate Spread Pulse Modulation in LPWANs for IoT applications}

\author{
Alexei V. Nikitin \\ Nonlinear LLC \\ Wamego, Kansas, USA \\ E-mail: avn@nonlinearcorp.com
}

\author{
Ruslan L. Davidchack \\ Sch. of Mathematics and Actuarial Sci., \\ U. of Leicester, Leicester, UK \\ E-mail: rld8@ leicester.ac.uk
}

\begin{abstract}
In low-power wide-area networks (LPWANs), various trade-offs among the bandwidth, data rates, and energy per bit have different effects on the quality of service under different propagation conditions (e.g. various types of fading), interference scenarios, multi-user requirements, and design constraints. Such compromises, and the manner in which they are implemented, further affect other technical aspects, such as system's computational complexity and power efficiency. At the same time, this difference in trade-offs also adds to the technical flexibility in addressing a broader range of the Internet of Things (IoT) applications. This paper addresses a physical layer LPWAN approach based on the Aggregate Spread Pulse Modulation (ASPM) and provides a brief assessment of its properties in an additive white Gaussian noise (AWGN) channel. In the binary ASPM, the control of the quality of service is performed through the change in the spectral efficiency, i.e., the data rate at a given bandwidth. Implementing M-ary encoding in ASPM further enables controlling service quality through changing the energy per bit (in about an order of magnitude range) as an additional trade-off parameter. Such encoding is especially useful for improving the ASPM's energy per bit performance, thus increasing its range and overall energy efficiency, and making it more attractive for use in LPWANs.

Index Terms-Aggregate spread pulse modulation (ASPM), intermittently nonlinear filtering (INF), Internet of things (IoT), LoRa, low-power wide-area network (LPWAN), M-ary ASPM (MASPM), physical layer (PHY), spread spectrum.
\end{abstract}

\section{INTRODUCTION}

In the Aggregate Spread Pulse Modulation (ASPM) [1, 2], the information is encoded in the amplitudes $A_{j}$ and/or the "arrival times" $k_{j}$ of the pulses in a digital "pulse train" $\hat{x}[k]$ with only relatively small fraction of samples having non-zero values:

$$
\hat{x}[k]=\sum_{j} \llbracket k=k_{j} \rrbracket A_{j},
$$

where $k$ is the sample index, $k_{j}$ is the sample index of the $j$-th pulse, $A_{j}$ is the amplitude of the $j$-th pulse, and the double square brackets denote the Iverson bracket [3]

$$
\llbracket P \rrbracket=\left\{\begin{array}{cc}
1 & \text { if } P \text { is true } \\
0 & \text { otherwise }
\end{array},\right.
$$

where $P$ is a statement that can be true or false. The average "pulse rate" $f_{\mathrm{p}}$ in such a train is $f_{\mathrm{p}}=F_{\mathrm{s}} / N_{\mathrm{p}}$, where $F_{\mathrm{s}}$ is the sample rate, and $N_{\mathrm{p}}=\left\langle k_{j}-k_{j-1}\right\rangle$ is the average interpulse interval. Note that for $N_{\mathrm{p}} \gg 1$ the pulse rate is much smaller than the Nyquist rate. Also note that for $N_{\mathrm{p}} \gg 1$ this train has a large peak-to-average power ratio (PAPR) even when $\left|A_{j}\right|=$ const, and is generally unsuitable for use as a modulating signal.
However, the designed pulse train $\hat{x}[k]$ given by (1) can be "re-shaped" by linear filtering:

$$
x[k]=(\hat{x} * \hat{g})[k]=\sum_{j} A_{j} \hat{g}\left[k-k_{j}\right],
$$

where $\hat{g}[k]$ is the impulse response of the filter and the asterisk denotes convolution. The filter $\hat{g}[k]$ can be, for example, a lowpass filter with a given bandwidth $B$. If the filter $\hat{g}[k]$ has a sufficiently large time-bandwidth product (TBP) $[4,5]$, most of the samples in the reshaped train $x[k]$ will have non-zero values, and $x[k]$ will have a much smaller PAPR than the designed sequence $\hat{x}[k]$. Such low-PAPR signal can then be used for modulating a carrier. If the combination of the amplitude $A_{j}$ and the arrival time $k_{j}$ of a pulse provides $M$ distinct "states," each pulse can encode $\log _{2} M$ bits, and the raw bit rate $f_{\mathrm{b}}$ in such a train is $f_{\mathrm{b}}=f_{\mathrm{p}} \log _{2} M$. Such signaling is commonly referred to as "M-ary." When $B \gg f_{\mathrm{b}}=\left(F_{\mathrm{s}} / N_{\mathrm{p}}\right) \log _{2} M$, it results in a low-rate message encoded in a wideband waveform, and thus ASPM is a spread-spectrum technique.

To keep the energy per bit low, we may prefer not to use the magnitudes of the pulses for encoding, only their polarities (i.e., keep $\left|A_{j}\right|=$ const) and the arrival times, since those can be changed without changing the energy of the pulses. For example, for the arrival times in (1) one can use

$$
k_{j}=j N_{\mathrm{p}}+\Delta k\left[m_{j}\right] \text {, }
$$

where $m_{j} \leq M$ is a positive integer and $\Delta k[m]$ is an integervalued invertible function, such that $0 \leq \Delta k[m]<N_{\mathrm{p}}$ and $\Delta k[m] \neq \Delta k[l]$ for $m \neq l$. Then for $m_{j} \in\{1,2, \ldots, M\}$ and $A_{j}=$ const the pulse train given by (1) encodes $\log _{2} M$ bits per pulse. We will refer to such M-ary encoding with $A_{j}=$ const as "unipolar."

Another bit can be added by using $A_{j}=(-1)^{a_{j}}$, where $a_{j}$ is either " 0 " or " 1 ," and we will refer to such signaling as "bipolar." Then for bipolar M-ary signaling equation (1) can be rewritten as

$$
\hat{x}[k]=\sum_{j} \llbracket k=j N_{\mathrm{p}}+\Delta k\left[m_{j}\right] \rrbracket(-1)^{a_{j}},
$$

where $m_{j} \in\{1,2, \ldots, M / 2\}$ and $a_{j} \in\{0,1\}$. Fig. 1 illustrates a designed pulse train with such bipolar M-ary encoding according to (5), with $\Delta k\left[m_{j}\right]=m_{j}-1$. In this example, $N_{\mathrm{p}}=9$ and for $m_{j} \leq N_{\mathrm{p}}$ each pulse can have $M=2 N_{\mathrm{p}}=18$ distinct states.

For a given designed pulse sequence $\hat{x}[k]$, the spectral, temporal and amplitude structures of the reshaped train $x[k]$ 


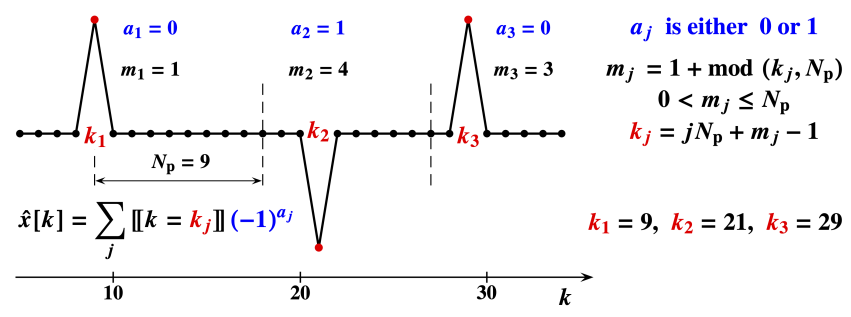

Figure 1. Example of designed pulse train with bipolar M-ary encoding.

will be determined by the choice of $\hat{g}[k]$. In particular, it may be desirable to select a filter $\hat{g}[k]$ that minimizes the PAPR of $x[k]$. Note that if the time duration of $\hat{g}[k]$ extends over multiple interpulse intervals, the instantaneous amplitudes and/or phases [6] of the resulting waveform are no longer representative of individual pulses. Instead, they are a "piled-up" aggregate of the contributions from multiple "stretched" pulses.

The key property of the large-TBP pulse shaping filter (PSF) $\hat{g}[k]$ is that its autocorrelation function (ACF), i.e., the convolution of $\hat{g}[k]$ with its matched filter $g[k]=\hat{g}[-k]$, has a much smaller TBP, in particular, sufficiently smaller than the ratio $B / f_{\mathrm{p}}$. Then, after demodulation and analog-to-digital (A/D) conversion in the receiver, the encoded binary sequence can be recovered by filtering with $g[k]$ and sampling the resulting pulse train at $k=j N_{\mathrm{p}}+\Delta k[m]$, where $m \in\{1,2, \ldots, M / 2\}$ for bipolar, and $m \in\{1,2, \ldots, M\}$ for unipolar encoding (i.e., using $g[k]$ as a decimation filter).

A good choice for the PSF would be a pulse that combines a small TBP of its ACF (e.g., close to that of a Gaussian pulse) with ACF's compact frequency support. An example would be a raised-cosine (RC) filter [7, e.g] with unity roll-off factor. The minimum required (Nyquist) sample rate for such a filter will be double its (baseband) physical bandwidth $B$, and the sample rate $F_{\mathrm{s}}$ can be expressed as $F_{\mathrm{s}}=2 N_{\mathrm{s}} B$, where $N_{\mathrm{s}} \geq 1$ is the oversampling factor. To minimize the power consumption, the memory usage, and the computational complexity of the digital processing, it may be beneficial to keep the sample rate in the transceivers designed for IoT applications as low as possible, i.e., to use $N_{\mathrm{s}}=1$. Through the rest of the paper, we will assume sampling with the Nyquist rate $F_{\mathrm{s}}=2 B$.

Since for a given designed pulse sequence $\hat{x}[k]$ the temporal and amplitude structures of the reshaped train $x[k]$ are determined by the PSF $\hat{g}[k]$, these structures can be substantially different even for the PSFs with the same ACF. As discussed in [1, 2], one can construct a great variety of large-TBP PSFs $\hat{g}_{1}[k]$, $\hat{g}_{2}[k]$, and so on, with the same small-TBP ACF $w[k]$, so that $\left(\hat{g}_{i} * g_{i}\right)[k]=w[k]$ for any $i$, while the convolutions of any $\hat{g}_{i}(t)$ with $g_{j}(t)$ for $i \neq j$ (cross-correlations) have large TBPs. Further, this property will also effectively hold for the PSFs $\hat{h}_{i}[k]$ such that $\hat{h}_{i}[k]$ is the discrete Hilbert transform of $\hat{g}_{i}[k]$, i.e., $\hat{h}_{i}[k]=H\left\{\hat{g}_{i}[k]\right\}[8,9]$. Therefore, using various combinations of PSFs $\hat{g}[k] \in\left\{\hat{g}_{1}[k], \hat{g}_{2}[k], \ldots\right\}$ and $\hat{h}[k] \in\left\{\hat{h}_{1}[k], \hat{h}_{2}[k], \ldots\right\}$, we can design different coherent and noncoherent modulation schemes with emphasis on particular spectral and/or temporal properties of the modulated signal.

\section{A. Binary ("one bit per pulse") encoding}

For example, in [10] we describe single-sideband, constantenvelope coherent and noncoherent ASPM configurations that use the "equidistant" designed train

$$
\hat{x}[k]=\sum_{j} \llbracket k=j N_{\mathrm{p}} \rrbracket(-1)^{b_{j}}
$$

to encode the binary sequence $\left(b_{1} b_{2} \ldots b_{j} \ldots\right)$. The raw bit rate $f_{\mathrm{b}}$ in such a train is $f_{\mathrm{b}}=F_{\mathrm{s}} / N_{\mathrm{p}}$, where $F_{\mathrm{s}}$ is the sample rate and $N_{\mathrm{p}}$ is the number of samples between pulses. We further show that, predictably, for an additive white Gaussian noise (AWGN) channel the uncoded bit error rate (BER) $P_{\mathrm{b}}$ of these binary ASPM configurations can be expressed as

$$
\begin{gathered}
P_{\mathrm{b}}=\frac{1}{2} \operatorname{erfc}\left(\sqrt{\frac{E_{\mathrm{b}}}{N_{0}}}\right)=\frac{1}{2} \operatorname{erfc}\left(\sqrt{\frac{N_{\mathrm{p}} \Gamma}{2}}\right) \quad \text { (coherent), } \\
P_{\mathrm{b}}=\frac{1}{2} \exp \left(-\frac{E_{\mathrm{b}}}{2 N_{0}}\right)=\frac{1}{2} \exp \left(-\frac{N_{\mathrm{p}} \Gamma}{4}\right) \quad \text { (noncoherent), }
\end{gathered}
$$

where $\operatorname{erfc}(x)$ is the complementary error function [11], $E_{\mathrm{b}}$ is the energy per bit, $N_{0}$ is the (one-sided) power spectral density (PSD) of the noise, and $\Gamma$ denotes the signal-to-noise ratio (SNR) defined as $\Gamma=\left(E_{\mathrm{b}} / N_{0}\right) \times\left(f_{\mathrm{b}} / B\right)$. Thus, at a given bandwidth, in the binary ASPM the control of the quality of service is performed through the change in the interpulse interval $N_{\mathrm{p}}$, i.e., the data rate.

\section{M-ARY VARIANTS OF ASPM}

In the binary ASPM, each pulse encodes one bit, hence the energy per bit $E_{\mathrm{b}}$ and the energy per pulse $E_{\mathrm{p}}$ are equal to each other, $E_{\mathrm{b}}=E_{\mathrm{p}}$. By encoding $\log _{2} M$ bits per pulse with the same energy, the energy per bit is reduced to $E_{\mathrm{b}}=E_{\mathrm{p}} / \log _{2} M$. Such encoding is especially useful for improving the ASPM's energy per bit performance, thus increasing its range and overall energy efficiency, and making it more attractive for use in LPWANs for IoT applications.

\section{A. Single-sideband M-ary ASPM with constant-envelope pulses}

For example, Fig. 2 illustrates a single-sideband M-ary ASPM link which uses constant-envelope transmitted pulses and is suitable for both coherent ('co') and noncoherent ('nc') detection.

In the designed pulse train $\hat{x}[k]$ according to (5), we use 8 distinct possible pulse locations relative to $j N_{\mathrm{p}}$ (see Fig. 2(I)). This train is then filtered with $\hat{g}[k]$ and $\hat{h}[k]$ to form the shaped trains $x_{g}[k]$ and $x_{h}[k]$. After digital-to-analog (D/A) conversion, $x_{g}(t)$ and $x_{h}(t)$ are used for quadrature amplitude modulation of a carrier with frequency $f_{\mathrm{c}}$, providing the transmitted waveform $x_{g}(t) \sin \left(2 \pi f_{\mathrm{c}} t\right)+x_{h}(t) \cos \left(2 \pi f_{\mathrm{c}} t\right)$ (Fig. 2(II)). If $\hat{g}[k]$ and $\hat{h}[k]$ are, say, the real and imaginary parts, respectively, of a nonlinear chirp with the desired ACF, e.g.

$$
\hat{g}[k]+i \hat{h}[k]=\llbracket 0 \leq k<n \rrbracket \exp (i \Phi[k]),
$$

where $\Phi[k]$ is the phase, then this waveform will occupy only a single sideband with the physical bandwidth $B$ equal to the baseband bandwidth of the chirp. In addition, if the pulses do not overlap (e.g., $N_{\mathrm{p}}>n+\max _{m}(\Delta k[m])$ ), this waveform will consist of constant-envelope pulses. 

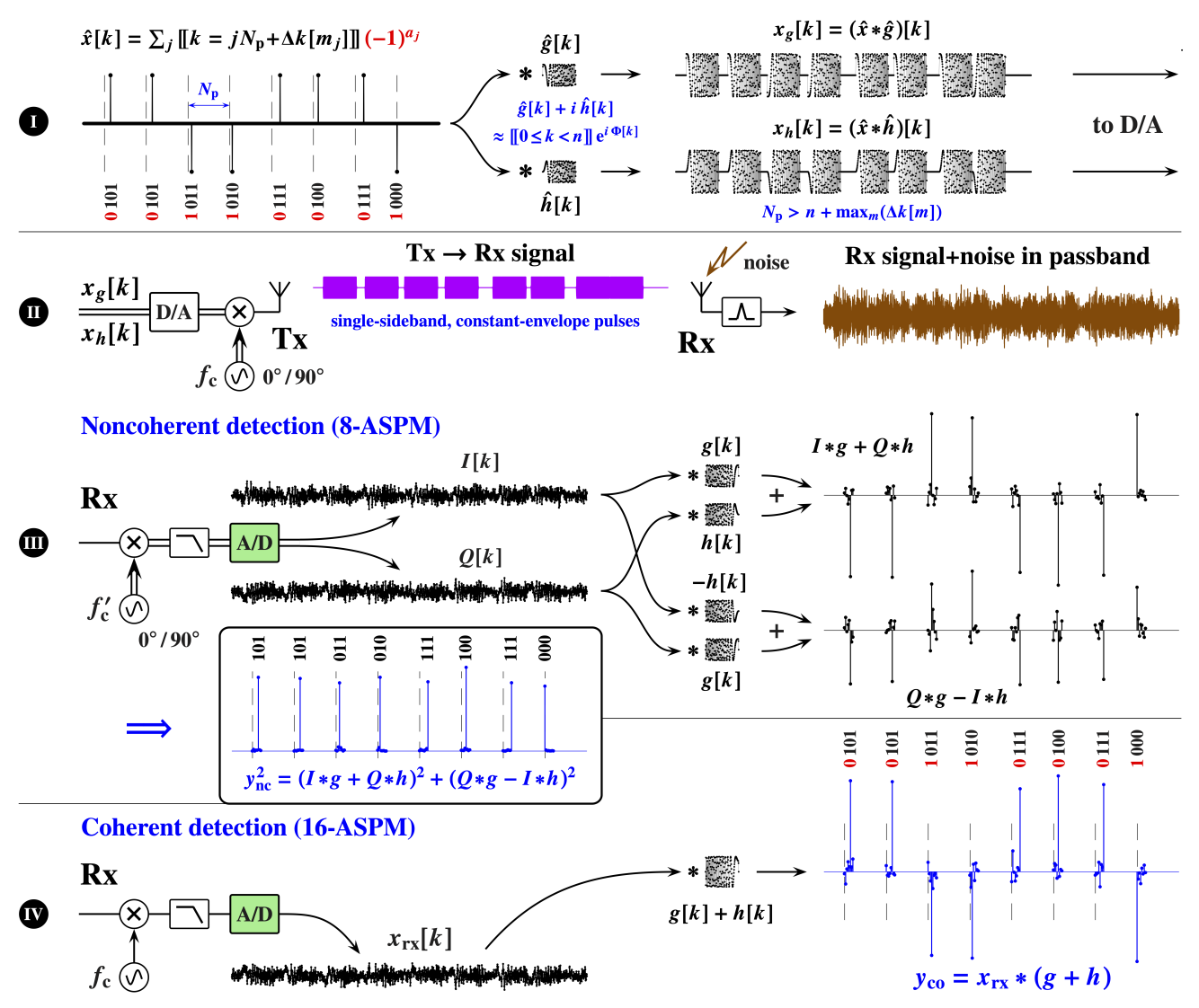

Figure 2. Illustration of single-sideband M-ary ASPM link with constant-envelope pulses and their noncoherent and coherent detection.

In the receiver, we can use either noncoherent or coherent detection. While demodulation techniques may vary, Figs. 2(III) and 2(IV) provide particular examples.

For noncoherent detection (Fig. 2(III)), in the receiver's (Rx) quadrature demodulator the noisy passband signal is multiplied by the orthogonal sinusoidal signals from a local oscillator, lowpassed, and converted to the in-phase and quadrature digital signals $I[k]$ and $Q[k]$. We then use the matched filters $g[k]$ and $h[k]$, as shown in Fig. 2(III), to obtain 8 samples per pulse of the high-peakedness pulse train $y_{\mathrm{nc}}[k]$ corresponding to the designed pulse train. Out of each 8 samples, the position of the sample with the largest magnitude will correspond to the position of the respective pulse in the designed train.

Noncoherent detection does not require precise carrier synchronization, neither in phase nor frequency, but it does not recover the polarity of the pulses. Thus we do not obtain the most significant bit in the symbols encoded in the pulses of the designed train. For coherent detection, we would need to recover the phase of the carrier. But then we can also measure, for each pulse, the polarity of the sample with the largest magnitude, and thus obtain an extra bit per pulse.

For coherent detection (Fig. 2(IV)), after multiplication by $\sin \left(2 \pi f_{\mathrm{c}} t+\pi / 4\right)$, lowpass filtering, and A/D conversion in the receiver, the resulting signal $x_{\mathrm{rx}}[k]$ is filtered with $g[k]+h[k]$ to form the bipolar baseband pulse train $y_{\mathrm{co}}=x_{\mathrm{rx}} *(g+h)$ corresponding to the designed train $\hat{x}[k]$.
Without loss of generality, the ACFs of $\hat{g}[k]$ and $\hat{h}[k]$ can be normalized to have the peak magnitudes equal to unity. Then, to avoid the interpulse interference in both coherent and noncoherent detection, we can require that

$$
w[\Delta k[m]-\Delta k[l]]=v^{2}[\Delta k[m]-\Delta k[l]]=\llbracket m=l \rrbracket,
$$

where $w=\frac{1}{2}(\hat{g} * g+\hat{h} * h)$ and

$$
v^{2}=\frac{1}{4}\left[(\hat{g} * g+\hat{h} * h)^{2}+(\hat{h} * g-\hat{g} * h)^{2}\right] .
$$

\section{B. Improving resistance to impulsive noise}

Note that the A/D conversion in the ASPM receiver can be combined with intermittently nonlinear filtering (INF) described in [12-14]. In INF, we establish a robust range that excludes noise outliers while including the signal of interest [15]. Then, we replace the outlier values with those in mid-range. Note that INF affects only a relatively small fraction of the samples in the signal+noise mixture, only those with exceedingly large magnitudes.

In Fig. 2, it is assumed that the A/D conversion can be performed in such a manner. As INF modifies the samples in the demodulated signal before the subsequent large-TBP filtering, its effect on the baseband signal of interest will be insignificant, while the contribution of the large-power noise outliers to the baseband noise will be reduced. This makes the link robust to outlier interferences, e.g. wideband impulsive noise commonly present in industrial environments [16], and increases 
the baseband SNR in the presence of such interferences. Since in the power-limited regime the channel capacity is proportional to the SNR, even relatively small increase in the latter will be beneficial.

\section{UNCODED BER PERFORMANCE OF M-ARY ASPM IN AWGN CHANNEL}

Let us initially provide a brief assessment of the M-ASPM properties in an AWGN channel. While AWGN is only a "background" noise component in the congested spectrum of most IoT applications, this assessment still provides a suitable benchmark for the subsequent evaluation of the M-ASPM performance under various more realistic propagation conditions and interference scenarios.

\section{A. Noncoherent M-ASPM}

Let us assume that we transmit the $j$-th pulse with $m_{j}=1$, and in the receiver sample at $j N_{\mathrm{p}}+\{\Delta k[1], \Delta k[2], \ldots, \Delta k[M]\}$. If $y_{m}^{2}=y_{\text {nc }}^{2}\left[j N_{\mathrm{p}}+\Delta k[m]\right]$, then the $j$-th symbol will be detected correctly when $y_{1}^{2}>\max \left\{y_{2}^{2}, y_{3}^{2}, \ldots, y_{M}^{2}\right\}$.

For AWGN with constant power density $N_{0}$, and in the absence of interpulse interference, $Y_{m}^{2}$ for $m>1$ can be viewed as independent and identically distributed (i.i.d.) variables having chi-square distribution with 2 degrees of freedom [11]. Thus the cumulative distribution function of the random variable $\mathcal{M}=\max \left\{Y_{2}^{2}, Y_{3}^{2}, \ldots, Y_{M}^{2}\right\}$ can be expressed as

$$
F_{\mathcal{M}}(x)=\left(1-\mathrm{e}^{-\frac{x}{2}}\right)^{M-1}=\sum_{k=0}^{M-1}(-1)^{k}\left(\begin{array}{c}
M-1 \\
k
\end{array}\right) \exp \left(-\frac{k}{2} x\right),
$$

where $\left(\begin{array}{l}n \\ m\end{array}\right)=\frac{n !}{(n-m) ! m !}$ is the binomial coefficient.

At the same time, $Y_{1}^{2}$ will have the noncentral chi-square distribution with 2 degrees of freedom and the noncentrality parameter $\lambda$ proportional to the peak power of the "ideal" pulse [11], and its cumulative distribution function can be expressed as

$$
F_{Y_{1}^{2}}(x)=1-Q_{1}(\sqrt{\lambda}, \sqrt{x}),
$$

where $Q_{1}(a, b)$ is the Marcum $Q$-function defined as the integral

$$
Q_{1}(a, b)=\int_{b}^{\infty} \mathrm{d} x \times \exp \left(-\frac{x^{2}+a^{2}}{2}\right) I_{0}(a x)
$$

for $a, b \geq 0$, and where $I_{0}(x)$ is the modified Bessel function of the first kind [17]. Therefore, the symbol error probability $P_{\mathrm{s}}$ can be expressed as

$$
\begin{aligned}
P_{\mathrm{S}}(\lambda) & =P\left(Y_{1}^{2}<\mathcal{M}\right)=\int_{0}^{\infty} \mathrm{d} x F_{Y_{1}^{2}}(x) \frac{\mathrm{d}}{\mathrm{d} x} F_{\mathcal{M}}(x) \\
& =1+\int_{0}^{\infty} \mathrm{d} x F_{\mathcal{M}}(x) \frac{\mathrm{d}}{\mathrm{d} x} Q_{1}(\sqrt{\lambda}, \sqrt{x}) .
\end{aligned}
$$

Evaluating the integral in the right-hand side of (14) by parts (see the Appendix), and noticing that the bit error probability $P_{\mathrm{b}}$ is related to the symbol error probability $P_{\mathrm{s}}$ as

$$
P_{\mathrm{b}}(\lambda)=\frac{M}{2(M-1)} P_{\mathrm{s}}(\lambda),
$$

leads to the following expression for $P_{\mathrm{b}}(\lambda)$ of noncoherent M-ASPM:

$$
P_{\mathrm{b}}(\lambda)=\frac{1}{2(M-1)} \sum_{k=2}^{M}(-1)^{k}\left(\begin{array}{c}
M \\
k
\end{array}\right) \exp \left(-\frac{k-1}{2 k} \lambda\right) .
$$

1) Value of noncentrality parameter $\lambda$ : The noncentrality parameter $\lambda$ is the ratio of the baseband peak signal power $A^{2}$ and the noise power $\sigma_{\mathrm{n}}^{2}, \lambda=A^{2} / \sigma_{\mathrm{n}}^{2}$, and it can be expressed in several different ways, for example as

$$
\lambda=\frac{2 E_{\mathrm{b}}}{N_{0}} \log _{2} M=\frac{2 \sigma_{\mathrm{c}}^{2}}{N_{0} f_{\mathrm{b}}} \log _{2} M=2 N_{\mathrm{p}} \frac{\sigma_{\mathrm{c}}^{2}}{N_{0} F_{\mathrm{s}}}=N_{\mathrm{p}} \Gamma,
$$

where $\sigma_{\mathrm{c}}^{2}$ is the power of the modulated carrier, thus describing the service quality in terms of different physical and numerical parameters of the link. In (17), as before, the SNR is defined as $\Gamma=\left(E_{\mathrm{b}} / N_{0}\right) \times\left(f_{\mathrm{b}} / B\right)$. Note that the spreading factor in the M-ASPM is $B / f_{\mathrm{b}}=N_{\mathrm{p}} /\left(2 \log _{2} M\right)$. Then, for example, in terms of the energy per bit $\gamma_{\mathrm{b}}=E_{\mathrm{b}} / N_{0}$, the bit error probability of noncoherent M-ASPM is

$$
P_{\mathrm{b}}\left(\gamma_{\mathrm{b}}\right)=\frac{1}{2(M-1)} \sum_{k=2}^{M}(-1)^{k}\left(\begin{array}{c}
M \\
k
\end{array}\right) \exp \left(-\frac{k-1}{k} \gamma_{\mathrm{b}} \log _{2} M\right) \text {. }
$$

Note that, for a given $\gamma_{\mathrm{b}}$, the bit error probability is a decreasing function of $M$ and, for $M \geq 64$, is the same as the bit error probability of noncoherent LoRa [18] with the spreading factor $\mathrm{SF}=\log _{2} M$. (Short for "Long Range," LoRa is a modulation technique derived from chirp spread spectrum (CSS), that has recently become one of the prevailing technologies in LPWANs for the IoT applications.) This is illustrated in Fig. 3, where the M-ASPM BER performance is compared with the respective performance of the noncoherent LoRa with different spreading factors. For LoRa, the BER approximation proposed in [19] is used, which is expressed as the product of the union bound on the bit error probability and a correction function.

In Fig. 4, the BER vs. SNR performance of the noncoherent 16-ASPM $\left(B / f_{\mathrm{b}}=N_{\mathrm{p}} / 8\right)$ is compared with the respective performances of the noncoherent LoRa with different spreading factors. As can be seen from the figure, in terms of the energy per bit performance for uncoded BER $=10^{-2}$ in an AWGN channel, the noncoherent 16-ASPM is approximately $60 \%$ to $80 \%$ as efficient as LoRa with the spreading factors ranging from $\mathrm{SF}=6$ to $\mathrm{SF}=12$.

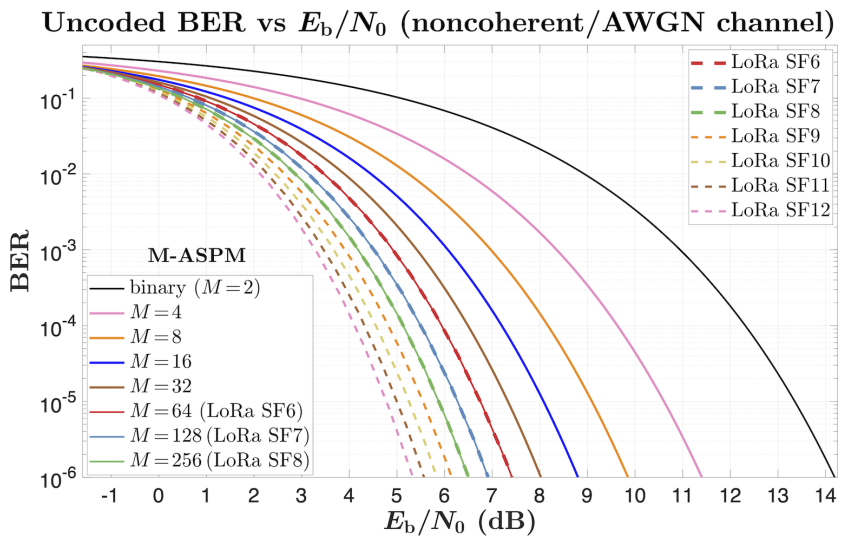

Figure 3. Uncoded BER vs $E_{\mathrm{b}} / N_{0}$ performances of LoRa (dashed lines) and single-sideband M-ASPM (solid lines) for noncoherent detection in AWGN channel. 


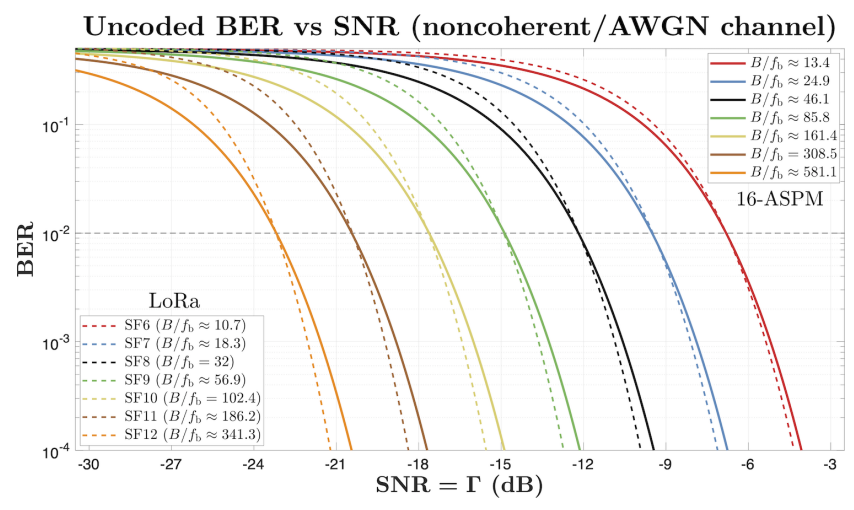

Figure 4. Uncoded BER vs SNR performances of LoRa (dashed lines) and single-sideband 16-ASPM (solid lines) for noncoherent detection in AWGN channel. For 16-ASPM, $B / f_{\mathrm{b}}=N_{\mathrm{p}} / 8$.

\section{B. $E_{\mathrm{b}} / N_{0}$ efficiency of coherent M-ASPM}

By using additional $M / 2$ distinct pulse locations in the binary coherent ASPM, each pulse can encode $m=\log _{2} M$ bits. For example, for $M=16$, the pulse train

$$
\hat{x}[k]=\sum_{j} \llbracket k=j N_{\mathrm{p}}+\left(4 a_{j}+2 b_{j}+c_{j}\right) n \rrbracket(-1)^{d_{j}},
$$

where $n$ is a nonzero integer, encodes a 4-bit sequence $\left(a_{1} b_{1} c_{1} d_{1} a_{2} b_{2} c_{2} d_{2} \ldots a_{j} b_{j} c_{j} d_{j} \ldots\right)$. To correctly identify a symbol in such M-ASPM, we need to correctly detect both the arrival time and the polarity of the pulse.

When the arrival time of a pulse with the peak magnitude $|A|$ is known, the probability of correctly detecting the polarity of this pulse in the presence of AWGN with zero mean and variance $\sigma_{\mathrm{n}}{ }^{2}$ can be expressed, using the complementary error function, as $\frac{1}{2} \operatorname{erfc}(-\mu)$, where $\mu=|A| /\left(\sigma_{\mathrm{n}} \sqrt{2}\right)$. We can further assume that $n$ in (19) is sufficiently large, and thus interpulse interference is negligible (e.g. $n \geq 2$ for coherent detection and pulse shaping with the ACF as an RC pulse with unity roll-off factor). Then, for a pulse train with the peak magnitude of the pulses equal to $|A|$, and $m=\log _{2} M$ bits per pulse encoding, the bit error probability can be expressed as

$$
P_{\mathrm{b}}(\mu)=\frac{M}{2(M-1)}\left[1-\frac{1}{2} \operatorname{erfc}(-\mu) P\left(\left|X_{1}\right|>\mathcal{M}\right)\right],
$$

where $X_{1}$ is a normal random variable with mean $\mu \propto|A|$ and variance $1 / 2$, and

$$
\mathcal{M}=\max \left\{\left|X_{2}\right|,\left|X_{3}\right|, \ldots,\left|X_{\frac{M}{2}}\right|\right\},
$$

where $X_{i}, i \in\{2,3, \ldots, M / 2\}$, are i.i.d. normal variables with zero mean and variance $1 / 2$.

For $Y=\left|X_{1}\right|$, its cumulative distribution function is that of the folded normal distribution, which can be expressed as

$$
F_{Y}(x ; \mu)=\frac{1}{2}[\operatorname{erf}(x+\mu)+\operatorname{erf}(x-\mu)]
$$

for $x \geq 0$. Then the probability to correctly detect the arrival time of the pulse is

$$
\begin{aligned}
& P\left(\left|X_{1}\right|>\mathcal{M}\right)=\int_{0}^{\infty} \mathrm{d} x\left[F_{Y}(x ; 0)\right]^{\frac{M}{2}-1} \frac{\mathrm{d}}{\mathrm{d} x} F_{Y}(x ; \mu) \\
& =\int_{0}^{\infty} \mathrm{d} x[\operatorname{erf}(x)]^{\frac{M}{2}-1}\left\{\frac{1}{\sqrt{\pi}}\left[\mathrm{e}^{-(x+\mu)^{2}}+\mathrm{e}^{-(x-\mu)^{2}}\right]\right\} .
\end{aligned}
$$

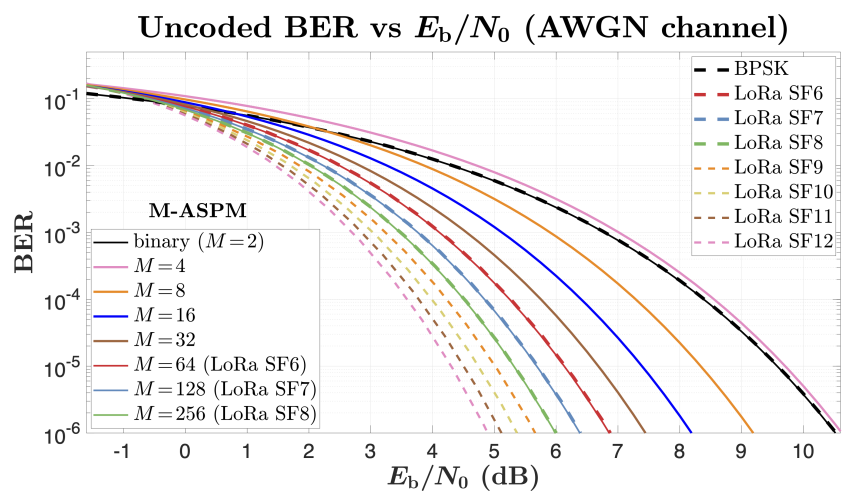

Figure 5. Uncoded BER vs $E_{\mathrm{b}} / N_{0}$ performances of BPSK and LoRa (dashed lines), and single-sideband M-ASPM (solid lines) for coherent detection in AWGN channel. For $M \geq 64$, M-ASPM $E_{\mathrm{b}} / N_{0}$ efficiency is that of LoRa with spreading factor $\mathrm{SF}=\log _{2} M$.

For $\mu=0$ the right-hand-side integral is equal to $2 / M$, and for $\mu>0$ it can be easily evaluated numerically.

1) Value of $\mu$ : For coherent detection, the ratio of the baseband peak signal power $A^{2}$ and the noise power $\sigma_{\mathrm{n}}^{2}$ is the same as for noncoherent detection [10], and thus $\mu=|A| /\left(\sigma_{\mathrm{n}} \sqrt{2}\right)=\sqrt{\lambda / 2}$, where $\lambda$ is the noncentrality parameter of the noncoherent ASPM given by (17). Then, for example,

$$
\mu=\sqrt{\frac{E_{\mathrm{b}}}{N_{0}} \log _{2} M}=\sqrt{\frac{N_{\mathrm{p}} \Gamma}{2}},
$$

where $\Gamma=\left(E_{\mathrm{b}} / N_{0}\right) \times\left(f_{\mathrm{b}} / B\right)$ is the SNR. The bit rate $f_{\mathrm{b}}$ is related to the pulse rate $f_{\mathrm{p}}$ as $f_{\mathrm{b}}=f_{\mathrm{p}} \log _{2} M$, and, as before, the spreading factor in the M-ASPM is $B / f_{\mathrm{b}}=N_{\mathrm{p}} /\left(2 \log _{2} M\right)$.

Figure 5 shows computed uncoded BER vs $E_{\mathrm{b}} / N_{0}$ performances of BPSK and LoRa (dashed lines), and single-sideband M-ASPM (solid lines) for coherent detection in an AWGN channel. Note that, just like in the noncoherent case, for $M \geq 64$ the M-ASPM $E_{\mathrm{b}} / N_{0}$ efficiency equals that of LoRa with the spreading factor $\mathrm{SF}=\log _{2} M$ [19].

In Fig. 6, the BER vs. SNR performance of the coherent 16-ASPM $\left(B / f_{\mathrm{b}}=N_{\mathrm{p}} / 8\right)$ is compared with the respective performances of the coherent LoRa with different spreading

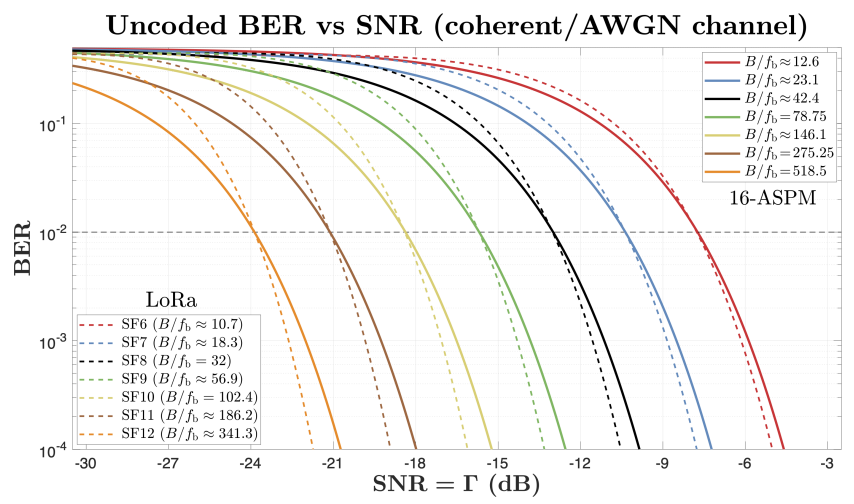

Figure 6. Uncoded BER vs SNR performances of LoRa (dashed lines) and single-sideband 16-ASPM (solid lines) for coherent detection in AWGN channel. For $16-\mathrm{ASPM}, B / f_{\mathrm{b}}=N_{\mathrm{p}} / 8$. 
Uncoded BER vs $E_{\mathrm{b}} / N_{0}$ (coherent/AWGN channel)

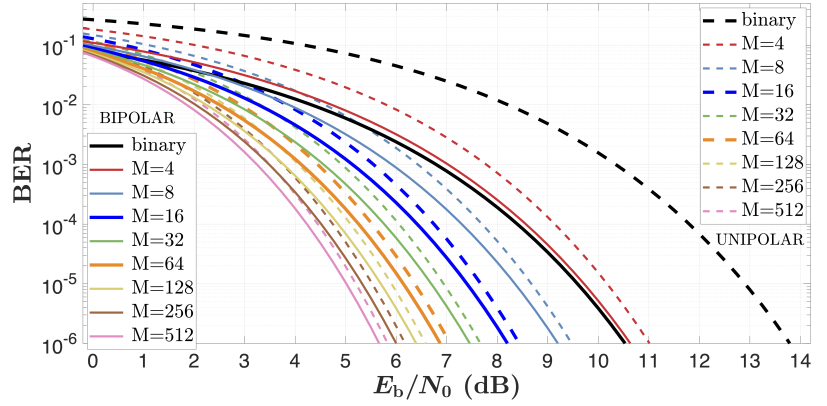

Figure 7. Comparison of uncoded AWGN BER vs $E_{\mathrm{b}} / N_{0}$ performances of unipolar (dashed lines) and bipolar (solid lines) signaling in coherent M-ASPM.

factors. As can be seen from the figure, in terms of the energy per bit performance for uncoded BER $=10^{-2}$ in an AWGN channel, the coherent 16-ASPM is approximately $70 \%$ to $90 \%$ as efficient as LoRa with the spreading factors ranging from $\mathrm{SF}=6$ to $\mathrm{SF}=12$.

2) Unipolar signaling: For both noncoherent and coherent M-ASPM, bipolar encoding requires only $M / 2$ distinct pulse locations. In comparison with the unipolar signaling, this doubles the maximum achievable data rate for a given $M$. However, the noncoherent detection always requires obtaining $M$ samples per pulse, and has identical energy per bit and computational efficiencies for either bipolar or unipolar encoding. In contrast, after the synchronization has been obtained, the detection for bipolar encoding in coherent M-ASPM requires sampling at only $M / 2$ data points per pulse, thus halving the per-bit computational intensity of numerical processing. In addition, the bipolar signaling in coherent M-ASPM has the advantage of higher AWGN energy per bit efficiency compared to the unipolar signaling.

Indeed, the bit error probability for the unipolar coherent detection can be expressed as

$$
\begin{aligned}
& P_{\mathrm{b}}^{\mathrm{up}}(\mu)=\frac{M}{2(M-1)}\left\{1-\int_{0}^{\infty} \mathrm{d} x[\operatorname{erf}(x)]^{M-1} \frac{\mathrm{d}}{\mathrm{d} x} F_{Y}(x ; \mu)\right\} \\
& =\frac{M}{4} \int_{0}^{\infty} \mathrm{d} x\left\{\frac{2}{\sqrt{\pi}} \mathrm{e}^{-x^{2}}\right\}[\operatorname{erf}(x)]^{M-2}[\operatorname{erf}(x+\mu)+\operatorname{erf}(x-\mu)],
\end{aligned}
$$

and it can be shown that $P_{\mathrm{b}}^{\mathrm{up}}(\mu ; M)>P_{\mathrm{b}}(\mu ; M)$ for $\mu>0$. This is illustrated in Fig. 7, that compares the uncoded AWGN BER vs. $E_{\mathrm{b}} / N_{0}$ performances of unipolar (dashed lines) and bipolar (solid lines) signaling in coherent M-ASPM. Predictably, the difference between $P_{\mathrm{b}}^{\mathrm{up}}(\mu ; M)$ and $P_{\mathrm{b}}(\mu ; M)$ becomes negligible in the limit of large $M$.

\section{SiMULATED BER VS SNR PERFORMANCE OF COHERENT AND NONCOHERENT 16 -ASPM}

Figure 8 compares the calculated (dashed lines) and the simulated (markers connected by solid lines) BERs for both coherent and noncoherent 16-ASPM links with different spreading factors $B / f_{\mathrm{b}}=N_{\mathrm{p}} / 8$.

For the coherent 16-ASPM, the designed pulse train $\hat{x}[k]$ is given by (19), where $n=2$. For the noncoherent 16-ASPM, the designed pulse train is

$$
\hat{x}[k]=\sum_{j} \llbracket k=j N_{\mathrm{p}}+\left(8 a_{j}+4 b_{j}+2 c_{j}+d_{j}\right) n \rrbracket,
$$

where $n=4$. In the transmitter, filtering $\hat{x}[k]$ with the PSF $\hat{g}[k]$ forms the modulating component $x_{\mathrm{I}}[k]$, and filtering $\hat{x}[k]$ with the PSF $\hat{h}[k]$ forms the modulating component $x_{\mathrm{Q}}[k]$. The ACF of $\hat{g}[k]$ is an RC pulse with unity roll-off factor. The filter $\hat{h}[k]$ approximates the discrete Hilbert transform of $\hat{g}[k]$, i.e., $\hat{h}[k] \approx H\{\hat{g}[k]\}[8,9]$, and thus $x_{\mathrm{Q}}[k]$ approximates the discrete Hilbert transform of $x_{\mathrm{I}}[k]$, i.e., $x_{\mathrm{Q}}[k] \approx H\left\{x_{\mathrm{I}}[k]\right\}$. Therefore, if after digital-to-analog conversion $x_{\mathrm{I}}(t)$ and $x_{\mathrm{Q}}(t)$ are used for quadrature amplitude modulation of a carrier with frequency $f_{\mathrm{c}}$, the resulting modulated waveform $x_{\mathrm{I}}(t) \sin \left(2 \pi f_{\mathrm{c}} t\right)+x_{\mathrm{Q}}(t) \cos \left(2 \pi f_{\mathrm{c}} t\right)$ effectively occupies only a single sideband with the physical bandwidth $B$ equal to the baseband bandwidth of $\hat{g}[k]$. Figure 9 shows both the PSFs $\hat{g}[k]$ and $\hat{h}[k]$ (left panel), and the PSD of the modulated carrier used in the simulations (right panel).

In the coherent receiver, the noisy passband signal is multiplied by the signal $\sin \left(2 \pi f_{\mathrm{c}} t+\pi / 4\right)$ from the local oscillator, lowpassed, and A/D converted to form the digital signal $x_{\mathrm{rX}}[k]$, which is then filtered with $g[k]+h[k]$ to form the baseband pulse train

$$
y_{\mathrm{co}}=x_{\mathrm{rx}} *(g+h) \text {. }
$$

For noncoherent detection, in the receiver's quadrature demodulator the noisy passband signal is multiplied by $\sin \left(2 \pi f_{\mathrm{c}} t+\varphi\right)$ and $\cos \left(2 \pi f_{\mathrm{c}} t+\varphi\right)$, lowpassed, and A/D converted to the in-phase (I) and quadrature $(\mathrm{Q})$ digital signals $I[k]$ and $Q[k]$. Then the received unipolar pulse train is formed as

$$
y_{\mathrm{nc}}^{2}=(I * g+Q * h)^{2}+(Q * g-I * h)^{2} .
$$

In the simulations, the bit error rates are determined by comparing the bit sequences extracted from the "ideal" transmitted signals (without noise), and from the transmitted signals affected by AWGN with a given PSD $N_{0}$.

\section{CONCLUSION}

In this paper, we demonstrate how M-ary signaling setup can be utilized within the ASPM to improve its energy per bit performance, increasing its range and overall energy efficiency and making it more attractive for use in LPWANs for IoT.

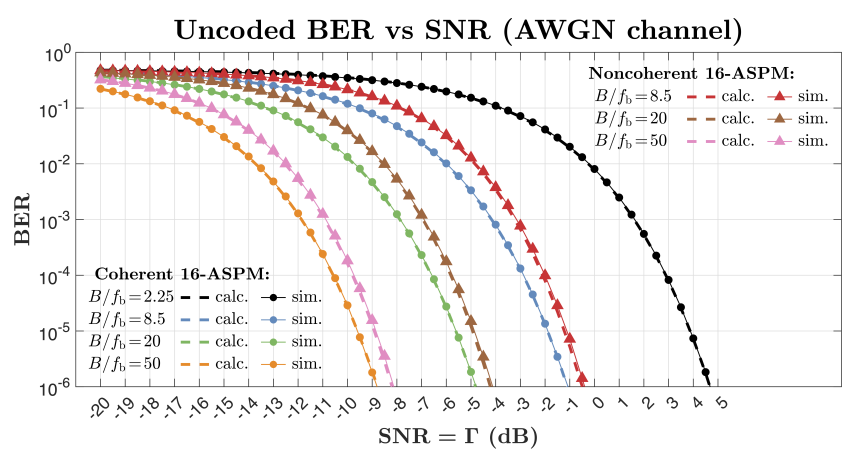

Figure 8. Calculated and simulated BERs as functions of AWGN SNRs for both coherent and noncoherent 16-ASPM with different values of $N_{\mathrm{p}}=8 \mathrm{~B} / f_{\mathrm{b}}$. 

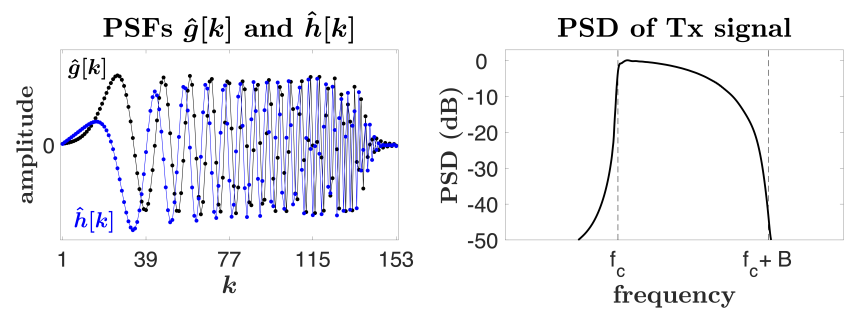

Figure 9. PSFs $\hat{g}[k]$ and $\hat{h}[k]$, and PSD of modulated carrier in simulations shown in Fig. 8.

Using various combinations of PSFs in ASPM, one can design numerous coherent and noncoherent modulations schemes with emphasis on particular spectral and/or temporal properties of the modulated signal. This allows us to accommodate different propagation conditions in different IoT environments, meet diverse multiuser and physical layer security requirements, and, overall, add to the technical flexibility in addressing a broader range of IoT applications, both static and mobile.

For example, in the constant-envelope link illustrated in Fig. 2, the time support of the pulse shaping filters is approximately equal to $n / F_{\mathrm{s}}$, and this link would be insensitive to the relative velocity $\Delta v$ between the transmitter and the receiver if $|\Delta v| / c \lesssim$ $F_{\mathrm{s}} /\left(n f_{\mathrm{c}}\right)$, where $c$ is the speed of light. Then, for the frequency ranges and bandwidths used by LoRa, a noncoherent ASPM link can be made insensitive to Doppler spreads in excess of several kilometers per second, which may be adequate for use in satellite IoT projects based on satellite constellations in low Earth orbit [20]. Further, the ASPM's equitable bandwidth and interpulse interval trade-off can be used to make the link resistant to the delay spreads below about $25 \mu \mathrm{s}(7.5 \mathrm{~km})$, while remaining tolerant to the Doppler spread above $200 \mathrm{~km} / \mathrm{h}$, accommodating most urban environments.

\section{APPENDIX}

Substituting (11) into (14), and considering a single term in the sum:

$$
\begin{aligned}
& \int_{0}^{\infty} \mathrm{d} x \exp \left(-\frac{k}{2} x\right) \frac{\mathrm{d}}{\mathrm{d} x} Q_{1}(\sqrt{\lambda}, \sqrt{x}) \\
& =-\frac{1}{2} \int_{0}^{\infty} \mathrm{d} x \exp \left(-\frac{\lambda+(k+1) x}{2}\right) I_{0}(\sqrt{\lambda x}) \\
& =-\frac{1}{k+1} \exp \left(-\frac{k}{2(k+1)} \lambda\right),
\end{aligned}
$$

where we have used the equalities $[17,21]$

$$
\frac{\mathrm{d}}{\mathrm{d} x} Q_{1}(\sqrt{\lambda}, \sqrt{x})=-\frac{1}{2} \exp \left(-\frac{\lambda+x}{2}\right) I_{0}(\sqrt{\lambda x}),
$$

$Q_{1}(a, 0)=1$, and $Q_{1}(a, \infty)=0$, the substitution $x=v^{2} /(k+1)$ in (A.1a), and the definition of the Marcum $Q$-function (13). Further,

$$
\begin{aligned}
P_{\mathrm{s}}(\lambda) & =1-\sum_{k=0}^{M-1} \frac{(-1)^{k}}{k+1}\left(\begin{array}{c}
M-1 \\
k
\end{array}\right) \exp \left(-\frac{k}{2(k+1)} \lambda\right) \\
& =\frac{1}{M} \sum_{k=2}^{M}(-1)^{k}\left(\begin{array}{c}
M \\
k
\end{array}\right) \exp \left(-\frac{k-1}{2 k} \lambda\right) .
\end{aligned}
$$

\section{REFERENCES}

[1] A. V. Nikitin and R. L. Davidchack, "Pulsed waveforms and intermittently nonlinear filtering in synthesis of low-SNR and covert communications," IEEE Access, vol. 8, pp. 173 250-173 266, 2020.

[2] A. V. Nikitin, "Method and apparatus for nonlinear filtering and for secure communications," US patent 11,050,591, June 29, 2021.

[3] D. E. Knuth, "Two notes on notation," American Mathematical Monthly, vol. 99, no. 5, pp. 403-422, May 1992.

[4] D. Gabor, "Theory of communication," Journal of the Institution of Electrical Engineers, vol. 93, no. 26, pp. 429-457, 1946.

[5] M. Vetterli and J. Kovačevic, Wavelets and subband coding. Prentice-Hall, 1995.

[6] B. Picinbono, "On instantaneous amplitude and phase of signals," IEEE Trans. Signal Process., vol. 45, no. 3, pp. 552-560, March 1997.

[7] J. G. Proakis and D. G. Manolakis, Digital signal processing: Principles, algorithms, and applications, 4th ed. Prentice Hall, 2006.

[8] R. N. Bracewell, The Fourier transform and its applications, 3rd ed. New York: McGraw-Hill, 2000.

[9] G. Todoran, R. Holonec, and C. Iakab, "Discrete Hilbert transform. Numeric algorithms," Acta Electroteh., vol. 49, no. 4, pp. 485-490, 2008

[10] A. V. Nikitin and R. L. Davidchack, "Aggregate spread pulse modulation in LPWANs for IoT applications," in 2021 IEEE 7th World Forum on Internet of Things (WF-IoT), New Orleans, LA, 14 June-31 July 2021.

[11] M. Abramowitz and I. A. Stegun, Handbook of Mathematical Functions. Dover, 1972.

[12] A. V. Nikitin and R. L. Davidchack, "Analog-domain mitigation of outlier noise in the process of analog-to-digital conversion," in Proc. IEEE Int. Conf. Commun. (ICC 2018), Kansas City, MO, 20-24 May 2018.

[13] — "Hidden outlier noise and its mitigation," IEEE Access, vol. 7, pp. 87873-87 886, 2019.

[14] — - "Complementary intermittently nonlinear filtering for mitigation of hidden outlier interference," in Proc. IEEE Military Commun. Conf. 2019 (MILCOM 2019), Norfolk, VA, 12-14 Nov. 2019.

[15] — - "Quantile tracking filters for robust fencing in intermittently nonlinear filtering," Preprint, http://arxiv.org/abs/1911.00736, 2019.

[16] J. Courjault, B. Vrigneau, O. Berder, and M. R. Bhatnagar, "How robust is a LoRa communication against impulsive noise?" in Proc. IEEE Int. Symp. on Personal, Indoor and Mobile Radio Commun. (PIMRC 2020), London, UK, 31 Aug.-3 Sept. 2020, pp. $1-6$.

[17] M. K. Simon, "The Nuttall $Q$-function: Its relation to the Marcum $Q$-function and its application in digital communication performance evaluation," IEEE Trans. Commun., vol. 50, no. 11, pp. 1712-1715, 2002.

[18] L. Vangelista, "Frequency shift chirp modulation: The LoRa modulation," IEEE Signal Process. Lett., vol. 24, no. 12, pp. 1818-1821, Dec 2017.

[19] G. Baruffa, L. Rugini, L. Germani, and F. Frescura, "Error probability performance of chirp modulation in uncoded and coded LoRa systems," Digital Signal Processing, vol. 106, p. 102828, 2020.

[20] A. A. Doroshkin, A. M. Zadorozhny, O. N. Kus, V. Y. Prokopyev, and Y. M. Prokopyev, "Experimental study of LoRa modulation immunity to Doppler effect in CubeSat radio communications," IEEE Access, vol. 7, pp. 75 721-75 731, 2019.

[21] Y. A. Brychkov, "On some properties of the Marcum Q function," Integral Transforms and Special Functions, vol. 23, no. 3, March 2012. 